\title{
A 36-year-old female from Kuwait with AIDS presenting with fever and abdominal pain
}

\author{
Keywords \\ acid-fast; AIDS; mycobacterium; \\ mycobactin $\mathrm{J}$.
}

\section{Case summary}

A 36-year-old woman from Kuwait with AIDS [CD4 cell count 43 cells $\mu l^{-1}$, human immunodeificency virus (HIV) viral load 70 copies $\mathrm{ml}^{-1}$ ] was transferred to our institution for evaluation and management of fever, abdominal pain, altered mental status, pancytopenia and generalized debility. She was treated for Pneumocystis jiroveci pneumonia, Cytomegalovirus encephalitis and Candida albicans fungaemia. Her medications included emtricitabine/tenofovir 200-300 mg once daily, raltegravir $400 \mathrm{mg}$ twice daily, trimethoprim-sulfamethoxazole $160-800 \mathrm{mg}$ orally every $48 \mathrm{~h}$, azithromycin $1250 \mathrm{mg}$ orally once weekly and valganciclovir $900 \mathrm{mg}$ twice daily. Initial laboratory data comprised a white blood cell count of $1.9 \times 10^{9} 1^{-1}$, neutrophil count of $1.5 \times 10^{9} 1^{-1}$, platelet count of $61 \times 10^{9} \mathrm{l}^{-1}$, haemoglobin $9.9 \mathrm{~g} \mathrm{dl}^{-1}$, aspartate transaminase $47 \mathrm{U} \mathrm{l}^{-1}$, alanine transaminase $59 \mathrm{U} \mathrm{l}^{-1}$, alkaline phosphatase $258 \mathrm{U} \mathrm{l}^{-1}$ and serum creatinine $0.2 \mathrm{mg} \mathrm{dl}^{-1}$. Computerized tomography of the abdomen showed moderate mesenteric and retroperitoneal lymphadenopathy without hepatosplenomegaly. A fine-needle aspiration biopsy of the mesenteric lymph node showed an extensive histocytic infiltrate containing innumerable acid-fast bacilli following Fite and Ziehl Neelsen acid-fast stains (Fig. 1a). The biopsy aspirate was plated onto Middlebrook 7H11//7H11 selective agar, and grew small, pinpoint, translucent colonies after

\section{Question}

This acid-fast organism grew after prolonged incubation and following Mycobactin J supplementation of the medium. What is the most likely aetiologic agent in this HIV-positive host?

\section{Answer options}

1. Mycobacterium avium complex

2. Mycobacterium genavense

3. Mycobacterium tuberculosis complex

4. Mycobacterium hemophilum

5. Mycobacterium leprae

6 weeks of incubation at $37{ }^{\circ} \mathrm{C}$ (Fig. 1b). A nucleic acid hybridization probe (Hologic GenProbe AccuProbe) specific for Mycobacterium avium complex (MAC) was negative. A definitive identification was made using 16S rRNA gene sequencing. The organism was also subcultured onto Mycobacterial Growth Indicator Tubes (MGIT; Becton Dickinson) with and without Mycobactin J supplementation. After 5 weeks of incubation at $37{ }^{\circ} \mathrm{C}$, the MGIT broth containing Mycobactin J produced a positive signal, whilst no growth was detected in the unsupplemented broth after 6 weeks.

\section{Discussion}

Correct answer: 2. Mycobacterium genavense.

The 16S rRNA gene sequence of the isolate was a $100 \%$ match to a $M$. genavense type strain. M. genavense is a fastidious, slow-growing, nontuberculous mycobacterium best known for causing opportunistic infections in patients with AIDS and in solid-organ transplant patients (Hoefsloot et al., 2013; Pechere et al., 1995). The clinical features of disseminated $M$. genavense resemble those of disseminated MAC infection (Thomsen et al., 1999). M. genavense rarely grows on conventional mycobacterial media (Coyle et al., 1992), but growth may be enhanced by the addition of Mycobactin J (an ironcontaining compound), or blood and charcoal, to acidified Middlebrook agar under microaerophilic conditions. In addition, avoidance of pre-treatment and extended incubation for up to 8 weeks may increase the detection of M. genavense (Thomsen et al., 1999). Colonies are typically smooth, pale yellow or white. In our patient, the original growth of $M$. genavense on solid medium in the absence of added Mycobactin J may have been due to the introduction of blood containing iron from the biopsy specimen. M. genavense isolates are generally susceptible to rifamycins, macrolides and fluoroquinolones and resistant to isoniazid. There is evidence of in vivo resistance to ethambutol and clofazimine (Thomsen et al., 1999). 


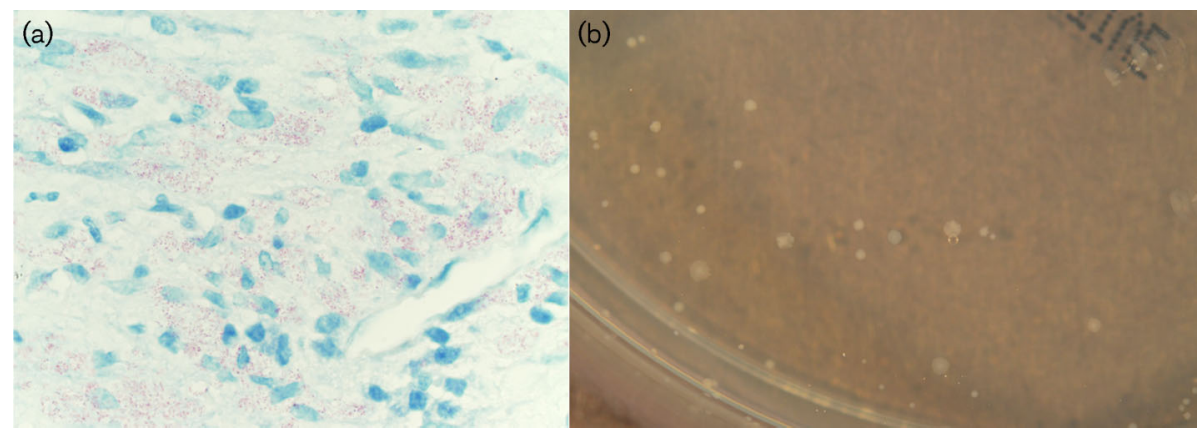

Fig. 1. (a) Fite stain of a mesenteric lymph node sampled by fine-needle aspiration biopsy showing histiocytic infiltrates containing many acid-fast bacilli. Magnification, $\times 400$. (b) Middlebrook agar plate with small, pinpoint, translucent colonies after 6 weeks of incubation at $37{ }^{\circ} \mathrm{C}$.

After MAC, M. genavense is the second most common non-tuberculous mycobacterium causing infection in AIDS patients, comprising $12.8 \%$ of all non-tuberculous mycobacterium infections in such patients (de Lastours et al., 2008). M. genavense can also produce infection in select immunosuppressed non-HIV-infected patients (Hoefsloot et al., 2013; Santos et al., 2014). It typically produces disseminated disease that can be similar to that of MAC and can frequently be recovered from blood, bone marrow, lymph nodes, stool, hepatic tissues and other organ tissues. M. genavense, however, is probably under-recognized due to difficulty in culture recovery of the organism. In our institution, this is the only case of $M$. genavense that we have encountered in the last 8 years. In samples from an appropriate immunological host with positive acid-fast bacillus smears but negative mycobacterial cultures, $M$. genavense should be included in the differential diagnosis, and the culture medium should be augmented with Mycobactin J and molecular diagnostics used to enhance diagnostic accuracy. In this case, the patient was treated initially with clarithromycin, ethambutol and rifabutin for presumed disseminated MAC infection. This treatment regimen was maintained after identification of $M$. genavense, and the patient is expected to continue therapy for at least 6 months after immune reconstitution (CD4 cell count $>100$ cells $\mathrm{mm}^{-3}$ ).

Poornima Ramanan ${ }^{1}$, Reeti Khare ${ }^{2}$, Pritish K. Tosh ${ }^{1}$, John W. Wilson ${ }^{1}$ and Nancy L. Wengenack ${ }^{2}$

${ }^{1}$ Division of Infectious Diseases, Mayo Clinic, Rochester, MN 55905, USA

${ }^{2}$ Division of Clinical Microbiology, Mayo Clinic, Rochester, MN 55905, USA

Correspondence: Nancy L. Wengenack (wengenack.nancy@mayo.edu)

\section{References}

Coyle, M. B., Carlson, L. C., Wallis, C. K., Leonard, R. B., Raisys, V. A., Kilburn, J. O., Samadpour, M. \& Bottger, E. C. (1992). Laboratory aspects of "Mycobacterium genavense," a proposed species isolated from AIDS patients. J Clin Microbiol 30, 3206-3212. de Lastours, V., Guillemain, R., Mainardi, J. L., Aubert, A., Chevalier, P., Lefort, A. \& Podglajen, I. (2008). Early diagnosis of disseminated Mycobacterium genavense infection. Emerg Infect Dis 14, 346-347.

Hoefsloot, W., Van Ingen, J., Peters, E. J., Magis-Escurra, C., Dekhuijzen, P. N., Boeree, M. J. \& Van Soolingen, D. (2013). Mycobacterium genavense in the Netherlands: an opportunistic pathogen in HIV and nonHIV immunocompromised patients. An observational study in 14 cases. Clin Microbiol Infect 19, 432-437.

Pechere, M., Opravil, M., Wald, A., Chave, J. P., Bessesen, M., Sievers, A., Hein, R., Von Overbeck, J., Clark, R. A. \& other authors (1995). Clinical and epidemiologic features of infection with Mycobacterium genavense. Swiss HIV Cohort Study. Arch Intern Med 155, 400-404.

Santos, M., Gil-Brusola, A., Escandell, A., Blanes, M. \& Gobernado, M. (2014).

Mycobacterium genavense infections in a tertiary hospital and reviewed cases in non-HIV patients. Patholog Res Int 2014, 371370.

Thomsen, V. O., Dragsted, U. B., Bauer, J., Fuursted, K. \& Lundgren, J. (1999).

Disseminated infection with Mycobacterium genavense: a challenge to physicians and mycobacteriologists. J Clin Microbiol 37, 3901-3905.

DOI 10.1099/jmmcr.0.003459 http://jmmcr.sgmjournals.org 